RELATIONSHIP BETWEEN ENVELOPE STRUCTURE AND ENERGY SOURCE OF NON-THERMAI MOTIONS

Hiroyasu Ando

Tokyo Astronomical Observatory, University of Tokyo

Mitaka, Tokyo, Japan 181

Abstract

The ionization zone in the envelope of the late type stars is reasonably considered as a heat engine to transform some of the radiative energy into mechanical energy. This idea is suggestive for explaining Linsky and Haisch's (1979) observation, which shows the sharp division into solar-type and non-solar type stars in the outer atmosphere. Also non-thermal velocity fields in "microturbulence" and in Wilson-Bappu effect are proposed to be formed essentially from this engine. Therefore, their envelope structure dependence observationally obtained is possibly explained by the envelope parameters $\left(g, T_{e}\right)$ dependence of the generated mechanical energy flux in this layer. If "microturbulence" is not contaminated by the other surface activities, it is expected to show a clear relation with envelope parameters ( $g, \mathrm{~T}_{e}$ ) similar to Wilson-Bappu effect.

AN ANALYSIS OF MICROTURBULENCE IN THE ATMOSPHERE OF THE F-TYPE SUPERGIANT GAMMA CYGNI

\author{
A.A. Boyarchuk and I.S. Lyubimkov \\ The Crimean Astrophysical Observatory \\ P/O Nauchny, Crimea, 334413, U.S.S.R.
}

Abstract

We have analysed high dispersion spectra of the supergiant $\gamma$ CYg (F8 Ib). On the basis of the curve of growth method it has been shown that there is no dependence of microturbulent velocity $\xi_{t}$ on excitation potentials of spectral lines. Using model atmospheres we considered about $100 \mathrm{Fe} I$ lines and found that no constant value $\xi_{t}$ makes possible to remove the systematic discrepancy in iron abundance between groups of lines with different equivalent widths. The depth dependence of microtur- 
bulence in the atmosphere is investigated. It is shown that parameter $\xi_{t}$ increases outwards from about $0-1 \mathrm{~km} / \mathrm{s}$ at the optical depth $\tau_{5000}=0.2$ to $10 \mathrm{~km} / \mathrm{s}$ at the depth $\tau_{5000}=10^{-3}$. Deduced function $\xi_{t}\left(\tau_{5000}\right)$ gives the same ixon abundance $\log \varepsilon(\mathrm{Fe})=$ $7.45 \pm 0.05$ for all groups of Fe I lines. The detailed analysis will be published in Izv. Crimean Astrophys. Obs.

\title{
THE SOLAR CHROMOSPHERIC MICROTURBUIENCE \\ AND \\ THE EMISSION OBSERVED AT ECLIPSE
}

\author{
Y. Cuny \\ Observatoire de Paris \\ 92190 Meudon, France
}

Abstract

For a long time there was not a clear interpretation of the fact that the emission observed at eclipse is systematically too high compared with predictions based on disk observations. This discrepancy has been attributed to the hydrogen density, the atomic abundances, or the multiplet excitation temperatures, the physical cause being assumed to be some inhomogeneities. We have shown (Liege 1978, under press) that it can be attributed to the microturbulence. The radiative energy absorbed in the doppler width of the absorption profiles of lines, produces high excitation temperatures. The relative abundances of Ba II, Sr II, Ti II with these excitation temperatures are in agreement with the photospheric abundances.

Assuming photospheric abundances, we get the hydrogen density versus the altitude while the observations give $n_{e} n_{p} / T_{e}{ }^{3 / 2}$. The solution of these two equations, taking account of non-LTE for hydrogen but without the assumption of hydrostatic equilibrium, gives a chromospheric model. The model obtained is very near the usual quiet chromospheric models based on disk data.

This work confirms the validity of our assumption of the influence of microturbulence on the chromospheric emission observed at eclipse. 